\title{
The tragic fate of group 3 innate lymphoid cells during HIV-1 infection
}

\author{
Xiaohuan Guo ${ }^{1}$ and Yang-Xin Fu \\ ${ }^{1}$ Tsinghua University School of Medicine, Beijing, China. ${ }^{2}$ Department of Pathology and Committee on Immunology, The University of Chicago, Chicago, Illinois, USA.
}

\begin{abstract}
HIV-1 infection usually leads to systemic chronic inflammation that is associated with gut microbial translocation. The recently defined group 3 innate lymphoid cells (ILC3s) are critical for maintenance of intestinal barrier function; however, it is not clear whether and how HIV-1 infection influences the function of these cells. In this issue of the JCl, Zhang and colleagues present compelling evidence that the survival and function of ILC3s are dramatically impaired by HIV-1 infection. The authors provide evidence that HIV-1 infection induces persistent activation of plasmacytoid dendritic cells ( $p D C s$ ) and production of type I IFNs, which together increase expression of death receptor CD95 on ILC3s and thereby promote subsequent ILC3 apoptosis. Together, these results identify a mechanism that explains the impaired intestinal barrier function that results from chronic HIV-1 infection and shed light on the role of pDCs in HIV-1 immunopathogenesis and therapy.
\end{abstract}

\section{Intestinal barrier dysfunction} after HIV-1 infection

Chronic HIV-1 infection is characterized by systemic inflammation, disruption of $\mathrm{T}$ lymphocyte homeostasis, and immune deregulation. It is widely accepted that chronic inflammation in HIV-1 patients is mainly due to translocation of intestinal microbes and/or their products from the gastrointestinal tract into the systemic circulation $(1,2)$. Normally, the intestinal mucosa serves as a barrier to microbes and their products. The gut barrier consists of three major components: (a) biological and microbial barriers, which are responsible for colonization resistance and regulation of the host immune response; (b) the physical barrier, which consists of gut epithelial cells and is maintained by tight junctions; and (c) the immune barrier, which includes various immune factors, immune cells, and gut-associated lymphoid tissues and mediates protection from microbial invasion (3). All of these barriers are rapidly altered in response to HIV-1 infection. HIV-1-associated changes include the dysregulation of gut flora (4), increased epithelial cell apoptosis (5), altered tight junction expression (6), depletion of intestinal $\mathrm{CD} 4^{+} \mathrm{T}$ cells, especially Th17 cells (7), and increased proinflammatory cytokines. Consequently, this barrier damage allows for microbial evasion, further driving systemic inflammation and promoting HIV-1 progression.

Innate lymphoid cells: key players in gut barrier function Innate lymphoid cells (ILCs) represent a recently recognized family of immune cells that have morphology and cytokine profiles similar to those of $\mathrm{T}$ cell lymphocytes, but lack rearranged antigen receptors. Within the past few years, three different ILC populations have been characterized in both mice and humans, including group 1 ILCs, group 2 ILCs, and group 3 ILCs (ILC3s) (8). Despite the relatively small

Related Article: p. 3692

Conflict of interest: The authors have declared that no conflict of interest exists.

Reference information: J Clin Invest. 2015;125(9):3430-3432. doi:10.1172/JCI83823.

size of these populations compared with $\mathrm{T}$ cells, ILC3s are enriched in lymphoid tissues and gut mucosal areas. Moreover, these cells have been shown to play essential roles in mucosal homeostasis by preventing pathogen infection and promoting tissue repair. ILC3s can produce IL-17 and IL-2 upon IL-1 $\beta$ and IL-23 stimulation and are the major producers of IL-22, which is essential for the gut barrier function in the intestine and associated lymphoid tissues (9). Previous studies have shown that naive ILC3- or IL-22-deficient mice have altered gut flora, reduced expression of tight junction proteins, mucins, and antimicrobial peptides, and increased rates of epithelial cell apoptosis $(9,10)$. Additionally, ILC3s can regulate adaptive immune cell responses through the expression of GM-CSF (11), MHCII (12), and lymphotoxin (13). The impaired gut barrier function and low-grade systemic and intestinal inflammation in ILC3-deficient mice result in persistent translocation and systemic dissemination of gut flora (14) as well as an increased susceptibility to pathogen infection and inflammation (14-18).

\section{Disruption of ILC3s after HIV-1 infection}

Recent studies have shown that the number of ILC3s and production of IL-22 are reduced in the intestines of humans and nonhuman primates after HIV-1 or SIV infection, respectively $(19,20)$. However, due to the lack of a robust animal model, the causation and the underlying mechanism that link ILC3 depletion and HIV-1 pathogenesis are poorly understood. In this issue, Zhang and colleagues confirmed that ILC3s are depleted in both blood and gut tissues of HIV-1-infected patients and that this depletion correlates with HIV-1 disease progression (21). The authors developed a technique to further investigate how the number of ILC3s may influence HIV-1 pathogenesis. Specifically, human ILC3s were transferred into experimental murine models and suc- 


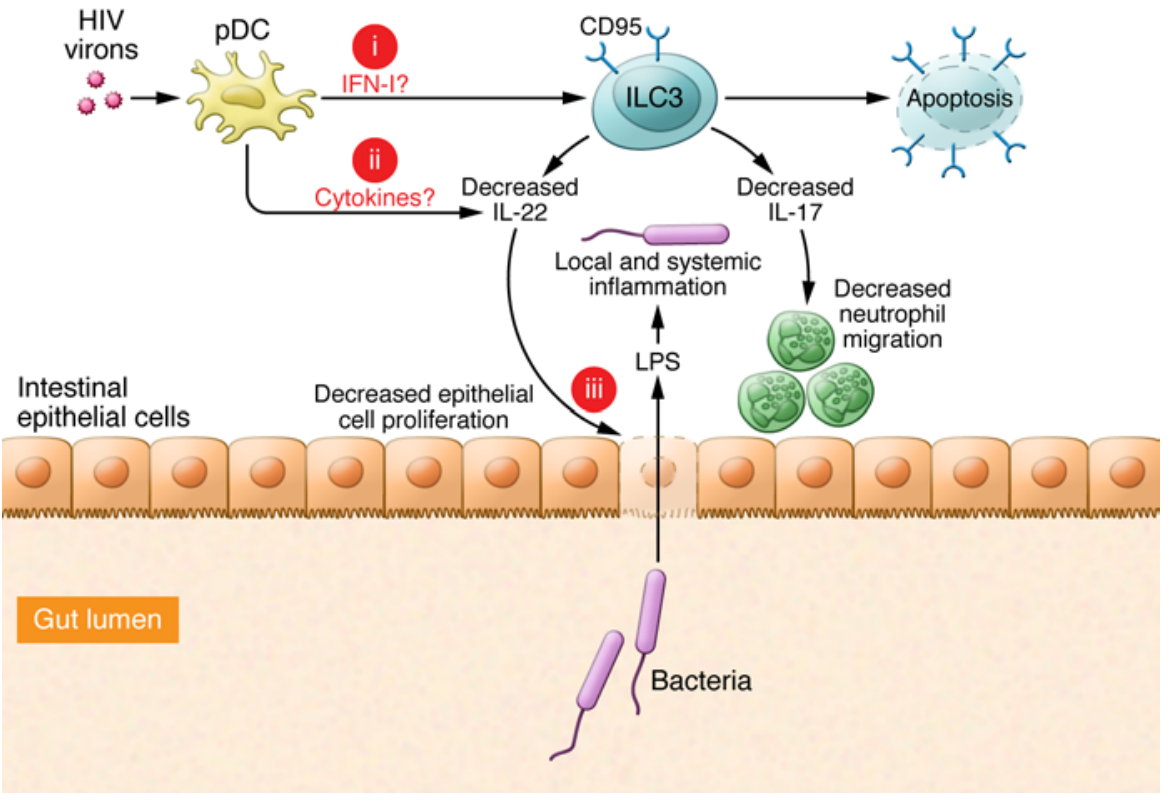

Figure 1. pDCs contribute to HIV-1-induced ILC3 dysfunction and depletion. HIV-1 infects and activates $\mathrm{pDCs}$, which produce high levels of IFN-I within lymph tissues. While increased IFN-I upregulates CD95 expression and sensitizes tissue-resident ILC3s to CD95/FasL-mediated apoptosis, it also partially impairs IL-17a and IL-22 production by gut ILC3s. Thus, the numeral and functional impairment of ILC3s may lead to the loss of intestinal epithelial integrity, resulting in the release of bacteria and their products, such as LPS, into blood that in turn induce systematic activation. However, three key questions require future study: (a) What is the role of IFN-I in the induction of ILC3 apoptosis in vivo? (b) Do pDCs produce other cytokines involved in the regulation of ILC3? (c) How do human ILC3s protect against mucosal bacterial infections and maintain the mucosal barrier? The resolution of these issues will help determine whether strategies aimed to modulate ILC3 responses have therapeutic potential to benefit patients with chronic HIV-1.

cessfully developed with normal phenotype and function within lymphoid organs of humanized animals. Persistent HIV-1 infection in these humanized mice led to the activation and depletion of human ILC3s in the spleen and mucosal lymph nodes. Moreover, HIV-1 infection affected the ability of human ILC3s to produce cytokines in infected mice. Interestingly, efficient suppression of HIV-1 replication with combined antiretroviral treatment (cART) restored human ILC3 levels in humanized mice. Together, these data indicate that HIV-1 infection disrupts the number and function of human ILC3s (Figure 1).

\section{pDC and type I IFNs: a double- edged sword in HIV-1 infection}

Whereas many cell types produce IFN- $\beta$ and other type I IFNs (IFN-I), plasmacytoid dendritic cells (pDCs) are the primary source of IFN- $\alpha$. Activated pDCs can produce large amounts of IFN-I, which in turn stimulate target cells through IFN- $\alpha / \beta$ receptor (IFNAR) signaling. pDCs have been reported to be the major producers of IFN-I during HIV-1 infection and are important for host defense against HIV-1 (22). Recent studies have also shown that pDCs contribute to immunopathology in some viral infections, including HIV-1 infection $(23,24)$, and in inflammatory bowel diseases (25). Zhang et al. determined that the number of ILC3s in HIV-1infected humanized mice negatively correlates with pDC activation and IFN-I production. pDC depletion and inhibition of IFN-I prevented ILC3 apoptosis and restored levels of human ILC3s after HIV-1 infection. Together, these data suggest that, although IFN-I suppress HIV-1 replication to a certain extent, these cytokines also induce pathogenesis through ILC3 depletion and subsequent intestinal barrier dysfunction during persistent HIV-1 infection (21). Using their humanized mouse model (22), Zhang and colleagues further demonstrated that chronic activation of pDCs and IFN-I in response to persistent HIV-1 infection upregulated expression of CD95 on human ILC3s. Importantly, CD95 was also elevated on
ILC3s isolated from peripheral blood and spleens of HIV-1-infected patients. Moreover, this increased expression of CD95 on human ILC3s likely enhances apoptosis and depletion of ILC3s during HIV-1 infection (21). Zhang and colleagues highlight an important role for pDCs and IFN-I in the depletion of ILC3s following HIV-1 infection. Additionally, the results of this study illustrate that these humanized mice can be used to study human ILC3s in vivo and suggest that therapeutic strategies that target pDCs or IFN-I have potential to restore ILC3 numbers and functions in HIV-1-infected patients (Figure 1).

\section{Perspectives}

Given the wide range of inflammatory conditions that lead to increased expression of IFN-I, the study by Zhang et al. suggests the need to further investigate whether ILC3s or other ILC populations are depleted through IFN-I or similar pathways in other chronic inflammation situations. Future studies should also address whether modulation of pDC and ILC3 responses has potential as a therapeutic strategy to provide a clinical benefit. It would be important to determine whether human ILC3s develop in the mucosal lamina propria of humanized mice or patient samples as well as when and how they are depleted by HIV-1 infection. As IL-22 is central to the maintenance of gut barrier function, the results of this study also raise the possibility that treatment with IL-22 could restore gut barrier function and minimize HIV-1 pathogenesis in infected patients.

\section{Acknowledgments}

This work was supported by NIH grant DK100427-01A1 (to Y.-X. Fu) and funds from the Center of Life Sciences, Tsinghua University (to X. Guo). We appreciate Joanna Wroblewska and Yuan Zhang's comments.

Address correspondence to: Xiaohuan Guo, Tsinghua University School of Medicine, Beijing 100084, China. Phone: 86.1062772171; E-mail: guoxiaohuan@ biomed.tsinghua.edu.cn. Or to: Yang-Xin $\mathrm{Fu}$, Department of Pathology and Committee on Immunology, The University of Chicago, Chicago, Illinois 60637, USA. Phone: 773.307.5478; E-mail: yfu@uchicago.edu. 
1. Miedema F, Hazenberg MD, Tesselaar K, van Baarle D, de Boer RJ, Borghans JA. Immune activation and collateral damage in AIDS pathogenesis. Front Immunol. 2013;4:298.

2. Brenchley JM, et al. Microbial translocation is a cause of systemic immune activation in chronic HIV infection. Nat Med. 2006;12(12):1365-1371.

3. Assimakopoulos SF, Dimitropoulou D, Marangos $\mathrm{M}$, Gogos CA. Intestinal barrier dysfunction in HIV infection: pathophysiology, clinical implications and potential therapies. Infection. 2014;42(6):951-959.

4. Gori A, et al. Early impairment of gut function and gut flora supporting a role for alteration of gastrointestinal mucosa in human immunodeficiency virus pathogenesis. JClin Microbiol.2008;46(2):757-758.

5. Epple HJ, et al. Acute HIV infection induces mucosal infiltration with $\mathrm{CD} 4^{+}$and $\mathrm{CD} 8^{+} \mathrm{T}$ cells, epithelial apoptosis, and a mucosal barrier defect. Gastroenterology. 2010;139(4):1289-1300.

6. Nazli A, et al. Exposure to HIV-1 directly impairs mucosal epithelial barrier integrity allowing microbial translocation. PLoS Pathog. 2010;6(4):e1000852.

7. Bixler SL, Mattapallil JJ. Loss and dysregulation of Th17 cells during HIV infection. Clin Dev Immunol. 2013;2013:852418.

8. Spits $\mathrm{H}$, et al. Innate lymphoid cells - a proposal for uniform nomenclature. Nat Rev Immunol. 2013;13(2):145-149.

9. Sonnenberg GF, Artis D. Innate lymphoid cells in the initiation, regulation and resolution of inflammation. Nat Med. 2015;21(7):698-708.

10. Artis D, Spits H. The biology of innate lymphoid cells. Nature. 2015;517(7534):293-301.

11. Mortha A, et al. Microbiota-dependent crosstalk between macrophages and ILC3 promotes intestinal homeostasis. Science. 2014;343(6178):1249288.

12. Hepworth MR, et al. Immune tolerance. Group 3 innate lymphoid cells mediate intestinal selection of commensal bacteria-specific CD4(+) T cells. Science. 2015;348(6238):1031-1035.

13. Kruglov AA, et al. Nonredundant function of soluble LTalpha3 produced by innate lymphoid cells in intestinal homeostasis. Science. 2013;342(6163):1243-1246.

14 . Sonnenberg GF, et al. Innate lymphoid cells promote anatomical containment of lymphoid-resident commensal bacteria. Science. 2012;336(6086):1321-1325.

15. Guo X, Liang Y, Zhang Y, Lasorella A, Kee BL, Fu YX. Innate lymphoid cells control early colonization resistance against intestinal pathogens through ID2-dependent regulation of the microbiota. Immunity. 2015;42(4):731-743.

16. Stefka AT, et al. Commensal bacteria protect against food allergen sensitization. Proc Natl Acad Sci U S A. 2014;111(36):13145-13150.

17. Qiu J, et al. Group 3 innate lymphoid cells inhibit T-cell-mediated intestinal inflammation through aryl hydrocarbon receptor signal- ing and regulation of microflora. Immunity. 2013;39(2):386-399.

18. Hazenberg MD, Spits H. Human innate lymphoid cells. Blood. 2014;124(5):700-709.

19. $\mathrm{Li} \mathrm{H}$, et al. Hypercytotoxicity and rapid loss of NKp44+ innate lymphoid cells during acute SIV infection. PLoS Pathog. 2014;10(12):e1004551.

20. Kim CJ, et al. A role for mucosal IL-22 production and Th22 cells in HIV-associated mucosal immunopathogenesis. Mucosal Immunol. 2012;5(6):670-680.

21. Zhang Z, et al. Plasmacytoid dendritic cells promote HIV-1-induced group 3 innate lymphoid cell depletion. J Clin Invest. 2015;125(9):3692-3703.

22. Zhang L, Su L. HIV-1 immunopathogenesis in humanized mouse models. Cell Mol Immunol. 2012;9(3):237-244

23. Li G, et al. Plasmacytoid dendritic cells suppress HIV-1 replication but contribute to HIV-1 induced immunopathogenesis in humanized mice. PLoS Pathog. 2014;10(7):e1004291.

24. Zhang L, Jiang Q, Li G, Jeffrey J, Kovalev GI, Su L. Efficient infection, activation, and impairment of pDCs in the BM and peripheral lymphoid organs during early HIV-1 infection in humanized rag2(-)/(-)gamma $\mathrm{C}(-) /(-)$ mice in vivo. Blood. 2011;117(23):6184-6192.

25. McNab F, Mayer-Barber K, Sher A, Wack A, O'Garra A. Type I interferons in infectious disease. Nat Rev Immunol. 2015;15(2):87-103. 